\title{
Caminos paralelos, deficiencias comunes: aproximación a los modelos públicos de recursos humanos en España y Portugal
}

\author{
Ramón Bouzas Lorenzo* \\ Enrique José Varela Álvarez"***
}

Palabras clave: España, Portugal, Administración pública, función pública, gestión de recursos bumanos.

Si algo propicia la participación en reuniones científicas que versan sobre una temática político-administrativa es la posibilidad de confrontar ideas con realidades a priori, supuestamente semejantes, mas dispares y heterogéneas en su fondo. Así lo hemos podido comprobar al abordar en uno de los foros más relevantes de nuestra profesión $^{1}$ una de las preocupaciones cardinales de la gerencia pública: la relativa a la función de personal, variable de enorme influencia en la operatividad y rendimiento de las organizaciones complejas.

Siendo conscientes de los límites que otros colegas advierten al afrontar estudios comparados (Parrado 2002: 218-266; Román y Láiz 2003), resolvimos adentrarnos en este proceloso territorio con el fin de contribuir a retratar las experiencias de dos Estados inmersos en procesos de homogeneización de numerosas prácticas de gestión, merced a las oportunidades creadas por la emergencia de nuevos espacios político-administrativos interregionales y, por tanto, con la ostensible inducción a través de instancias transnacionales de algunos de los mecanismos que permiten asemejar sus políticas de gestión pública.

Como sabemos, los sistemas de función pública -para ser más precisos, de empleo público- más comúnmente aceptados en el ámbito occidental se dividen entre los abiertos o de empleo, caracterizados por la flexibili-

\footnotetext{
"Profesor Titular de Ciencia Política y de la Administración de la Universidad de Santiago de Compostela

Profesor Ayudante de Ciencia Politica y de la Administración de la Universidad de Vigo.

${ }^{1}$ Nos referimos al panel Función de Recursos Humanos: ¿Estamos todavía en el punto de partida?, coordinado por el profesor Ramón Bouzas Lorenzo, organizado en el marco del X Congreso Internacional de sobre la Reforma y Administración del Estado (CLAD, Santiago de Chile, 1821 de octubre de 2005), en el que hubo ocasión de discutir las cuestiones clave de los sistemas de función pública de España (Administración Ge-
} neral del Estado y Comunidades Autónomas), Portugal, Colombia y México. 
dad, simplicidad y temporalidad, y los cerrados o de carrera, que destacan por su estabilidad, permanencia y complejidad (Martínez 1999: 14). Si bien los casos que se comentan en este trabajo difícilmente se ajustan a un perfil puro, a grandes rasgos, las modalidades de empleo público española y portuguesa ${ }^{2}$ aproximan sus características a un sistema de carrera, aunque con tendencia hacia el modelo de empleo.

Siendo la base jurídica común y, aun advirtiendo -como se confirmará en las próximas páginas- que las particularidades históricas, políticas y administrativas han surtido modelos cualitativa y cuantitativamente diferentes ${ }^{3}$, sus sistemas de gestión de recursos humanos han resultado relativamente estables, de evolución incremental, a pesar del devenir de las experiencias seguidas por cada Administración en materia de reforma o modernización administrativa.

Este es el contexto, de tronco común, pero de fuertes contrastes, de la función de personal en las Administraciones centrales de España y Portugal, que constituye el telón de fondo del presente trabajo, que pretende explorar las características de los subsistemas administrativos (Baena del Alcázar 2000) de ambos páses, extrayendo así conclusiones compartidas desde el análisis de los efectos de las medidas que sucesivamente se han ido implantando en su función de personal. Para ilustrar la dificultad de la comparación de ambas realidades', podemos servirnos de las palabras de Colomer (1995: 199-243) donde se pone de relieve esta situación: «Excepto en el breve y remoto período de unión de Portugal a la Corona española en 1581-1640, los pueblos y los gobernantes de los dos países de la península ibérica han seguido una larga tradición de ignorarse mutuamente. Tras la pérdida de la mayor parte de sus dominios coloniales y durante una gran parte de la historia contemporánea, España y Portugal han sufrido regímenes autoritarios y han permanecido cerrados al exterior. Incluso en sus relativamente escasas relaciones externas, tanto comerciales como culturales e informativas, España ha estado más comunicada con otros países de la Europa continental, y Portugal con Gran Bretaña, que los dos páises entre sír. El panorama se agrava en el caso de las instituciones y organizaciones públicas lusas, en lo relativo a sus espacios administrativos y, en particular, en aspectos más concretos como la función de personal, donde la ausencia de atención académica es clamorosa. Y es que resultan claros los límites en la medición de realidades supuestamente comunes (función pública según el modelo continental, con base en el derecho administrativo y evoluciones similares del Estado de bienestar) en dos países con modelos territoriales diversos (España, con descentralización política regional en 17 Comunidades Autónomas, y Portugal, con desconcentración administrativa regional, a excepción de Azores y Madeira, que cuentan con descentralización político-administrativa), con un peso cualitativa y cuantitativamente diferente de sus niveles de Gobierno (central-regional-local) y con un volumen de población distinta (cuatro a uno a favor de España) lo que, sin duda, incide en la prestación de servicios, la oferta de políticas públicas y en el número y tipología de empleados públicos que desarrollan su labor en dichas organizaciones públicas.

\section{Programas de modernización y expectativas sobre la Función de Personal de la AGE en España}

Los procesos de cambio que se han ido introduciendo en la Administración General del Estado española (en adelante, AGE) tienen su origen en las grandes transformaciones que desde el inicio de la transición, a fina-

\footnotetext{
${ }^{2}$ Aludimos siempre a los modelos de recursos humanos de las Administraciones centrales.

${ }^{3}$ Disponemos, por tanto, de modelos de organización particulares, en los que los empleados públicos, y sobre todo el funcionario, han desarrollado su labor de un modo peculiar y distintivo (derecho administrativo, jerarquía, interés general, etc.). Mas, no se trata sólo de desempeñar una labor dentro de una organización, de ser un subsistema, un factor más, sino de hacerlo dentro de un marco jurídico concreto, con limitaciones basadas en elementos objetivos (selección, desempeño, formación, promoción, retribuciones, negociación colectiva, responsabilidad, etc.), que la constriñen, y, por supuesto, con elementos subjetivos (cultura, principios y valores públicos), que la alejan de la caracterización de las organizaciones privadas (Ramió 1999: 1-30).

${ }^{4} \mathrm{La}$ escasez de trabajos realizados desde una perspectiva comparada, que aborden los modelos administrativos portugués y español, no impide observar las distancias que en la práctica muestran dos modelos de tradición común. Podríamos reseñar, entre otras referencias las aportaciones de González (1985), Román (1993), Colomer (1995), Cavero y Zamora (1996), González (1999) o Lagares (2000).
} 
les de los setenta, se producen como efecto de la transferencia de competencias desde la Administración central a las nacientes Administraciones autonómicas. A lo largo de los últimos treinta años no ha habido ningún otro proceso que en términos de redistribución del poder, riesgo político, efectos organizativos o magnitudes de los recursos comprometidos merezcan el calificativo de reforma.

Ahora bien, un macroproyecto de tales características, que redibuja el mapa administrativo español -piénsese que tras haber sido el referente por excelencia en la provisión de servicios generales, la AGE finaliza el siglo especializada en la prestación de servicios de carácter estratégico para el conjunto del Estado, acentuando sus funciones de planificación y coordinación, en detrimento de su actividad de gestión y atención directa a la ciudadanía - pronto mostraría aristas en las que resultaba ineludible una intervención más afinada, como ejemplifica la necesidad de disponer de un marco que homogeneizase y garantizase la coordinación de las políticas orientadas a la función pública o a la provisión de servicios públicos entre las autonomías, las corporaciones locales y la AGE.

Es así como nacen las modificaciones que desde inicio de los ochenta se producen en diversas normas básicas: en primer lugar, la Ley 30/1984, de medidas para la reforma de la función pública ${ }^{6}$, que transforma significativamente el modelo de función pública, situando en un punto central al puesto de trabajo, como clave de la ordenación y articulación del empleo público, de la carrera y del sistema retributivo; en segundo lugar, la normativa sobre procedimiento administrativo (enraizada en disposiciones de 1958), que contribuiría a renovar, a través de la Ley de régimen jurídico de las Administraciones públicas y del procedimiento administrativo común, el vínculo del ciudadano con la Administración y a clarificar competencialmente los ámbitos político y administrativo; y en tercer lugar, la promulgación de la Ley 6/97, de organización y funcionamiento de la AGE (LOFAGE), que instaura el principio de servicio a los ciudadanos y permite reorganizar/racionalizar la Administración tanto en su estructura nuclear como en su articulación periférica, regular el estatus funcional, administrativo y jurídico de la extensa malla de organizaciones del sector público e introducir medidas orientadas a la rendición de cuentas y al aumento de la autonomía de tales entidades.

Tras la reestructuración de cuerpos y la flexibilización de la función pública auspiciada por la Ley 30/84, tan importante para el proceso de descentralización autonómica, el verdadero punto de partida de la introducción de microiniciativas en materia de gestión de recursos humanos comienza en 1989 con la publicación de las «Reflexiones para la Modernización de la Administración del Estado» ${ }^{7}$, en las que se proponían actuaciones de toda índole justificadas en la necesidad de responder a las limitaciones del gasto público, la presión ciudadana; la realidad autonómica o el nuevo escenario de integración europea.

Como consecuencia de la vía abierta por las «Reflexiones», entre 1992 y 1994 se elaboran los estudios sobre la mejora de la eficacia, eficiencia y calidad de los servicios públicos que conducirán al Plan de Modernización de la Administración del Estado, un proyecto tan ambicioso (204 líneas de actuación) como impreciso, con el doble objetivo de mejorar la gestión interna de las organizaciones y aumentar la calidad de los servicios públicos. En síntesis, además de actuar sobre la estructura organizativa y los procedimientos, desde la perspectiva de la función de recursos humanos, el plan contenía un amplio conjunto de medidas, que giraban en torno a seis ejes

${ }^{5}$ Según el INAP (datos del año 1983) y el Registro Central de Personal (datos del año 2005), la Administración General del Estado pasa de los 1.357.000 empleados (80\% de todo el personal público del Estado) en 1983 a 540.868 (23\%) en 2005.

${ }^{6}$ Esta norma se completaría posteriormente con la Ley 53/1984, de incompatibilidades del personal al servicio de las Administraciones públicas y la Ley 9/1987, de órganos de representación, determinación de las condiciones de trabajo y participación del personal al servicio de las Administraciones públicas.

${ }^{7}$ Esta nueva concepción del cambio es resumida elocuentemente por A. Martín Acebes, Director General de Organización, Puestos de Trabajo e Informática del Ministerio para las Administraciones Públicas en 1991: «proponemos, por tanto, abandonar el concepto de reforma administrativa y sustituirlo por el de modernización de la Administración que se apoya en una estrategia gradualista y persistente de cambio» (MAP/Fundación Formación y Tecnología 1992: 30). 
de actuación: planificación de los recursos humanos (tomando como eje la Oferta de Empleo Público); selección de personal; formación; motivación; desconcentración de competencias y creación de un nuevo estilo directivo.

Tras esta iniciativa, será necesario esperar hasta 2000 para encontrar nuevamente una propuesta con ambición, pero de escasa originalidad frente a su antecedente: el Libro Blanco de la Modernización de la Administración (MAP 2000). En él, algunos de los temas presentados casi una década antes (cultura, liderazgo, evaluación de resultados, simplificación de procedimientos, motivación, selección, formación y desarrollo) se rinden a la retórica del capital humano, la sociedad del conocimiento, la calidad (mejora continua; benchmarking; EFQM) y la importancia de una actuación de acuerdo con un sólido código ético. El proyecto tendría mayor proyección práctica desde el plano de la servucción que desde la perspectiva de la función de personal, que, salvo por el hecho de reconocer -a través de la manifiesta reiteración de ideas- que se partía de un escenario de gestión poco más avanzado que el de 1991, el único elemento original que ofrecía era la exhortación a un urgente replanteamiento de la política de recursos humanos, que convertía la simplificación de la función pública (flexibilización; simplificación de cuerpos y escalas; impulso de la movilidad de funcionarios) y la mejora de los procesos selectivos (adaptación a necesidades; agilización; diversificación de las herramientas de selección) en los objetivos centrales del proyecto.

Hasta el momento, una exploración detenida del modelo de función de personal de la AGE no deja lugar a dudas sobre su anclaje a los sistemas más tradicionales de administración de personal ${ }^{8}$ y sobre la profusión de mejoras previstas todavía en suspenso.

El último capítulo que en el presente se desenvuelve en relación con la todavía irresuelta política de recursos humanos está incorporado a lo que el actual equipo responsable del Ministerio para las Administraciones Públicas en el Gobierno de Rodríguez Zapatero ha denominado «Decálogo para una Nueva Administración» ${ }^{9}$. Entre las diez medidas, que abarcan aspectos diversos de lo que nace con espíritu de convertirse en una nueva política de gestión pública -buscaría como fin último una mayor aproximación a la ciudadanía por medio de la consolidación de un patrón de gestión más integrado de los recursos humanos asociado a un fuerte desarrollo de la administración electrónica (proyecto «ciudadano.es»; proyecto «MAP en Red»)-, al margen de elementos de índole organizativa y operati$\mathrm{va}^{10}$, figuran varias propuestas vinculadas con los recursos humanos: elaboración de un nuevo estatuto de la función pública; diseño de un sistema de retribución con mayor dependencia de la evaluación del desempeño; mejora de las condiciones laborales (conciliación de la vida laboral y familiar; igualdad de oportunidades); renovación del proceso de selección (agilización del sistema; revaloración de los períodos de formación y prácticas); elaboración de un código ético dirigido a fomentar entre los empleados públicos la vocación de servicio; y dinamización de la comunicación con los representantes de los empleados públicos (Foro de Diálogo Social).

En algunos de los temas planteados se han dado los primeros pasos: la voluntad de pergeñar una actuación consensuada con los empleados se sustantivó en el Acuerdo para el Diálogo Social en las Administraciones Públicas (21 de septiembre de 2004), que propicia un proceso de negociación tendente reformar las normas que pre-

${ }^{8}$ A ellos aludía con evidente tono de desaliento en el inicio de la década de los noventa una importante responsable de la política de modernización: «ocuparse de los contratos laborales, el reclutamiento, la gestión de las nóminas y seguros sociales, la tramitación de vacaciones y permisos y, como mucho, el adiestramiento de los trabajadores en actividades estandarizadas o habilidades ligadas a su puesto de trabajo» (MAP/Fundación Formación y Tecnología 1992: 43).

${ }^{9}$ El proyectó se hizo público en el I Encuentro sobre la Modernización de la Administración pública llevado a cabo a finales de 2004.

${ }^{10}$ En términos organizativos, las principales actuaciones abarcan la elaboración de una Ley de Agencias públicas y Consorcios de cooperación interterritorial (redefinición de organismos públicos dotados de mayor autonomía de gestión y fomento del empleo de contratos de gestión por objetivos), la reforma de la Administración periférica (actualización normativa; renovación de medios y rediseño de servicios); la creación de una red de oficinas integradas; la reestructuración de servicios a través de una base divisional; y la infusión de una nueva cultura gerencial basada en el fomento de los valores de la evaluación de resultados. Desde el punto de vista operativo se pretende crear una Agencia de Evaluación de la Calidad de los Servicios; reforzar los mecanismos de coordinación (modernización de las inspecciones de servicios); intensificar la simplificación de procedimientos (proyecto Simplifica); y culminar la implementación del «Plan Conecta» (conjunto de actuaciones en gobierno electrónico) (MAP 2005b). 
cisa la mejora de las condiciones de trabajo de los empleados públicos; asimismo, el Consejo de Ministros aprobó el 4 de marzo de 2005 el Plan para la Igualdad de Género en la Administración General del Estado, que pretende afrontar un conjunto ambicioso de situaciones hasta ahora prácticamente desatendidas ${ }^{11}$; finalmente, con la finalidad de mejorar la cualificación de los empleados, ajustar la distribución territorial y funcional del personal y la carga de trabajo y adaptar los efectivos a las necesidades reales, se ha avanzado la posibilidad de introducir un plan de redistribución de personal y un proyecto de jubilaciones anticipadas e incentivadas de carácter voluntario a través de un Programa de Racionalización y Rejuvenecimiento de la AGE (MAP 2005a).

Sin menoscabo de las iniciativas relacionadas, por encima de cualquier otra pretensión, la elaboración de un nuevo estatuto de la función pública es el objetivo más ambicioso, el elemento clave del que depende el progreso futuro de la función de recursos humanos. Existe, en ese sentido, una firme voluntad de avanzar en su elaboración y, de hecho, con el horizonte de su aprobación durante el primer trimestre de 2007, a partir del documento base Informe sobre el Estatuto Básico del Empleado Público, elaborado por una comisión ministerial creada al efecto a finales de 2004 (INAP/Goberna 2005).

Además de contribuir a repensar una norma que tuvo sentido en el escenario del proceso de descentralización y transferencia de recursos a las Comunidades Autónomas durante los ochenta, y que ha demostrado poseer aspectos positivos (los valores de objetividad, imparcialidad, profesionalidad, mérito y capacidad que impregnan la actuación pública o la libre concurrencia y competitividad en el acceso al empleo público), la elaboración del Estatuto responde a múltiples necesidades: afrontar la heterogeneidad creciente del empleo público; renovar la legislación vigente (combatir su uniformismo y su excesiva rigidez y complejidad); evitar la sustitución del régimen jurídico de la función pública por el derecho laboral; atenuar la conflictividad competencial de los órganos involucrados en los procedimientos administrativos; aclarar la situación de las entidades públicas que disponen de regímenes jurídicos especiales; combatir el excesivo peso de la movilidad ascendente en la carrera profesional; y crear un marco que facilite la resolución de problemas entre Administraciones y que, a la vez, sea lo suficientemente flexible para permitir que cada entidad responda a sus circunstancias distintivas. Para ello, se pretende actuar sobre varias áreas problemáticas:

- Tipología del empleo público: definición de las funciones de los funcionarios; regulación del régimen jurídico y tipos de contratación laboral; combate de la temporalidad; aclaración de las situaciones de interinidad; determinación del estatus del personal eventual y directivo.

- Planificación de recursos humanos: flexibilización; oferta de empleo público; registros de personal; integración de sistemas de información; reordenación del trabajo (flexibilización).

- Selección: rediseño de los órganos de selección; agilización de los sistemas selectivos; diversificación de pruebas de ingreso; redefinición del acceso a posiciones de interinidad o temporales.

- Promoción: diseño de una nueva carrera administrativa articulada en torno al desempeño del puesto de trabajo y al desarrollo de las competencias de los empleados públicos; introducción de la evaluación del desempeño; definición de la carrera del personal laboral y de los mecanismos de promoción interna; reforzamiento de la política de formación continua.

\footnotetext{
${ }^{11}$ Entre estas medidas cabe destacar la flexibilización de horarios; la inclusión en los procesos de provisión de puestos de criterios basados en la valoración de obligaciones familiares (guarda legal de menores o atención a personas mayores); la tendencia a la paridad de género en los órganos de selección; el fomento de la presencia de la mujer en ámbitos de la Administración cuya presencia es deficitaria; el estímulo del acceso de la mujer a posiciones de mando intermedio y gerencia; la atenuación de la excesiva feminización de determinadas unidades; la reducción de la temporalidad en el empleo femenino; la impartición de actividades de formación continua dentro de la jornada laboral; y la tipificación del acoso sexual en el régimen disciplinario.
} 
- Provisión de puesto y movilidad: revisión del sistema de provisión de puestos; rediseño de la movilidad interna e interadministrativa; revisión del sistema de asignación de puestos y funciones y movilidad del personal laboral.

- Régimen administrativo y derechos básicos de los empleados públicos: unificación de situaciones funcionariales y laborales; definición de las situaciones de servicios especiales y excedencia, así como las causas de extinción del empleo público; nueva descripción de derechos básicos.

- Sistema retributivo: incorporación de factores de cualificación profesional, trabajo desempeñado y resultados obtenidos; reordenamiento del sistema retributivo del personal laboral.

- Cultura organizativa y ética: difusión de valores centrados en la orientación a la consecución de resultados y a la calidad de la prestación del servicio y atención al ciudadano; reforzamiento de la ética del servicio público.

- Dispositivos de representación, participación y negociación colectiva: reconsideración del ejercicio de representación colectiva; rediseño de órganos de participación; creación de órganos especializados en la negociación colectiva.

- Incompatibilidades: regulación; reforzamiento de la profesionalidad e imparcialidad de los directivos y altos cargos de las organizaciones.

- Régimen disciplinario: agilización del procedimiento sancionador; flexibilización del catálogo de sanciones y adaptación al sistema de promoción profesional y evaluación de los empleados.

- Coordinación entre Administraciones: reforzamiento de los dispositivos de coordinación.

El paso del tiempo confirmará si las iniciativas prosperan o, por el contrario, como ya ha sucedido en proyectos anteriores, quedan subordinadas a la consecución de las intervenciones complementarias -en este caso, dispositivos de administración electrónica- que lubrican todo el proceso ${ }^{12}$.

\section{Visión técnica de la Función de Personal}

La impresión de que desde inicios de los ochenta se detiene la evolución de la función de personal se refrenda, a grandes rasgos, al conocer las opiniones de los técnicos destinados a las unidades responsabilizadas de los recursos humanos.

Con la finalidad de actualizar algunas de las impresiones obtenidas a partir de los estudios de opinión realizados por el CIS hasta 1993 a los funcionarios ${ }^{13}$ y, al mismo tiempo, afinar la obtención de datos sobre la amplitud e importancia de la función de personal en la práctica, se entrevistó a responsables de unidades de recursos humanos situados bajo la supervisión de las Direcciones Generales de Recursos Humanos correspondientes o la dependencia de las Subsecretarías o Secretarías Generales Técnicas de cada ministerio. Los datos sobre la opinión de los

\footnotetext{
${ }^{12}$ Este artículo fue concluido antes de la aprobación definitiva por el Congreso, de la Ley del Estatuto Básico del Empleado Público, el día 29 de marzo de 2007 (publicada en el BOE de 13 de abril de 2007, entrando en vigor el día 13 de mayo de 2007), con lo que su contenido queda fuera del objeto de análisis del presente trabajo.

${ }^{13}$ Estudios n. ${ }^{\circ} 1359$ (CIS 1983); 1741 (CIS 1988); 1871 (CIS 1990) y 2054 (CIS 1993). Estas consultas del CIS exploraron las condiciones laborales de los empleados (autopercepción, prestaciones, carrera, retribución, actitudes directivas y sindicales) desde tres escenarios: el proceso de transferencias (especialmente de personal) a las Comunidades Autónomas; los cambios introducidos por la Ley de medidas para la reforma de la función pública y los programas de modernización ejecutados a lo largo de los noventa.
} 
técnicos se basan en la realización de entrevistas estructuradas a 18 directores y subdirectores generales de recursos humanos de los 16 ministerios de la AGE entre el 11 de mayo y el 8 de julio de 2005. Se trata de funcionarios de carrera pertenecientes a los grupos A y B, que se responsabilizan, con arreglo a lo establecido en la Ley 6/1997, de Organización y Funcionamiento de la AGE, de tareas que afectan al personal de su ministerio respectivo y sus organismos autónomos, entre otras: planificación, gestión y administración de los recursos humanos; elaboración de las relaciones de puestos de trabajo; gestión de las retribuciones; gestión de las relaciones con las organizaciones sindicales y asociaciones profesionales de funcionarios, así como de la negociación colectiva; elaboración de los planes de formación y mejora del desempeño del personal; dirección de la ejecución y tramitación, en el ámbito de su competencia, de los expedientes de compatibilidad y disciplinarios; planificación, dirección y gestión de la acción social y de los programas de prevención de la salud laboral; y coordinación e impulso de las estadísticas propias.

Respecto al perfil formativo, trayectoria y orientación profesional de los entrevistados, decir que se trata de un contingente mayoritariamente compuesto por individuos de entre 37 y 45 años, de formación jurídica, aunque carente de otros estudios de especialización en materia de función de personal que no sean los que con carácter generalista han recibido como parte de la formación estipulada para acceder a puestos directivos. La mayoría lleva entre 14 y 18 años trabajando en la Administración central -en general, no tienen antecedentes en otras organizaciones de los sectores público o privado-, cuentan con una experiencia media de 8 años en áreas que gestionan la función de recursos humanos, y llevan, casi todos ellos, menos de 5 años en su posición actual como responsables de personal.

El eje de las entrevistas giró en torno a la exploración de la carga habitual de tareas de las unidades encargadas de los recursos humanos; los efectos en la función de personal de las políticas de gestión pública llevadas a cabo durante los últimos veinte años; y el contenido de la práctica de recursos humanos en la AGE.

\section{a) Contenido de las Unidades de Recursos Humanos}

En coherencia con las previsiones que realiza la Ley 6/97, las tareas que mayor atención reclaman dentro del conjunto de las responsabilidades del puesto se centran en el cultivo de una gama limitada y rutinaria de labores propias de la administración de recursos humanos ${ }^{14}$ : provisión y modificación de puestos; contratación y supervisión de procesos selectivos; elaboración de notas informativas y proyectos; consignación de incidencias laborales; prevención de riesgos laborales o negociación con centrales sindicales.

En especial, en los departamentos cuya actividad carece de proyección transversal, resulta muy escaso el grado de influencia que desde las unidades responsabilizadas del personal se puede ejercer en las decisiones de las políticas que debe llevar a cabo cada ministerio. En consonancia, los técnicos de recursos humanos manifiestan su contrariedad al considerar que la unidad de la que son responsables no ocupe, en términos de importancia estructural y presupuestaria, el lugar que merece dentro de su organismo, y reconocen que una dotación de mayores medios humanos podría contribuir a mejorar su actuación.

\section{b) Modernización de la Administración}

En relación con las políticas de gestión pública emprendidas por la AGE, resulta revelador que los entrevistados declaren desconocer si en su organización se han llevado a cabo iniciativas de reforma y / o modernización administrativa. Un análisis más detallado de las respuestas apunta a que no es la presencia de diferencias semánticas entre

\footnotetext{
${ }^{14}$ Por razón de sobrecarga de trabajo, consideran que las unidades responsabilizadas de la función de personal no tienen por qué prestar atención a otras tareas de gestión.
} 
la concepción de las prácticas de reforma frente a las de modernización el motivo del reconocimiento de tal desconocimiento. Resulta, de hecho, significativo que sea la escasa antigüedad en el desempeño del presente cargo la característica común de quienes afirman desconocer la implantación de estas prácticas. Tal vez, el hecho de asociarlas a su posición actual determinaría la ignorancia de este particular -los entrevistados se autopercibirían situados en una dimensión de la gestión novedosa respecto a su trayectoria precedente, y metodológicamente cabría la posibilidad de haber inducido un error que llevaría a estos individuos a relacionar exclusivamente la reforma y la modernización con la práctica de recursos humanos-, pero que no dejaría de poner en tela de juicio el rendimiento de todas las acciones informativas que en su día se impulsaron con la finalidad de difundir dichos programas (lo cual, en último término, invitaría a preguntar quién es destinatario de facto del diseño de la estrategia de comunicación: la ciudadanía o los empleados). Quienes afirman tener información sobre la implantación de programas de reforma / modernización muestran en su mayoría insatisfacción no sólo con los resultados que en términos de gestión pública han suscitado tales iniciativas, sino que subrayan su escaso impacto en la política de gestión de personal -cuestionada de manera explíitia-. Para estas personas, las medidas ${ }^{15}$ que mayor frustración han provocado hasta la fecha son la simplificación normativa; la evaluación del desempeño y el rendimiento; la subcontratación de servicios y los intentos de cambio de cultura. Por el contrario, la incorporación de nuevas tecnologías de la información, las prácticas (técnicas) de acercamiento al ciudadano y la formación de directivos son las propuestas que proporcionan mayor satisfacción.

En el marco de los programas de reforma/modernización y desde el punto de vista de la política de personal, la mayoría de los encuestados considera que sus unidades han recibido el apoyo necesario para adaptarse a los cambios producidos, aunque enfatizan que un aumento de los recursos económicos (presupuesto) hubiese mejorado el resultado.

\section{c) Contenido de la función de recursos bumanos en el ámbito público}

Para conocer el alcance de la práctica de recursos humanos en el ámbito público se solicitó la respuesta de los responsables de personal a 22 preguntas que abarcaban desde la apreciación general de la importancia de algunas de las más destacadas dimensiones de la gestión y administración de recursos humanos hasta cuestiones concretas sobre el modo, frecuencia y finalidad con que se ejecutan algunas de las tareas (análisis de puestos; planificación; orientación; diagnóstico de clima y cultura; estudios de motivación; formación; evaluación del rendimiento) en que se han materializado algunos proyectos de modernización llevados a la práctica en experiencias de nuestro entorno, y que, además, han ocupado a nivel operativo un lugar destacado en la agenda de las iniciativas de mejora de la función de recursos humanos impulsadas por la AGE desde los años ochenta.

En primer lugar, de un listado de 26 actividades ligadas a la función de recursos humanos ${ }^{16}$, los entrevistados atribuyeron a ocho de ellas la categoría de «muy importante» en el desempeño de la responsabilidad de personal en el ámbito público: realización de análisis de puestos de trabajo; previsión de necesidades; diseño de pla-

${ }^{15}$ Las opciones que debían valorar eran: la simplificación de procedimientos; la simplificación de normativa; la incorporación de nuevas tecnologías de la información; la formación de empleados públicos; las prácticas de acercamiento al ciudadano; la formación de directivos; la evaluación del desempeño y rendimiento; la reorganización de estructuras organizativas; la descentralización de competencias en otros organismos públicos; la privatización de organismos públicos; la subcontratación de servicios; la reducción de niveles jerárquicos; el cambio de cultura organizativa y la difusión de prácticas de gobierno electrónico.

${ }^{16}$ Estas actividades son: realización de análisis de puestos de trabajo; previsión de necesidades de personal; diseño de planes y políticas de gestión de personal; programación y participación en tareas de reclutamiento y selección; actividades de orientación y acogida de los empleados; diseño organizativo; desarrollo de planes de carrera, promoción y movilidad; asistencia en problemas de rendimiento de los empleados; planificación de actividades de formación; control de inversión y gasto en personal; desarrollo de directivos; gestión de calidad; desarrollo del trabajo en equipo; evaluación del desempeño y rendimiento; control de nóminas; diseño de programas de motivación; relación con las fuerzas sindicales; intervención en problemas disciplinarios; análisis de cultura y clima; realización de estudios sobre la plantilla; diseño y aplicación de programas de seguridad higiene; diseño y mantenimiento sistema de información sobre personal; negociación de convenios; diseño y gestión del sistema de comunicación; análisis y gestión de la cultura organizativa y planificación de personal. 
nes y políticas de gestión de personal; planificación de actividades de formación; control de nóminas; relación con las fuerzas sindicales; realización de estudios sobre la plantilla; y diseño y aplicación de programas de seguridad y salud laboral. Sin embargo, sin llegar a imputar a ninguna de las restantes actividades una «importancia nula», relegaron a una posición muy secundaria entre sus prioridades a la supervisión de tareas vinculadas con el desarrollo de directivos; la intervención en problemas disciplinarios; la negociación de convenios; el diseño y gestión de sistemas de comunicación; y el análisis y gestión de la cultura organizativa. Más allá de la constricción de los comentarios a la que obliga la brevedad de la entrevista, en aclaraciones posteriores matizarían que la menor relevancia atribuida tendría su origen en la consideración de algunas de las actividades propuestas fuera del ámbito de la función de recursos humanos (comunicación; cultura) o al rechazo que infundirían en los técnicos por razones de complejidad (directivos) o conflictividad (régimen disciplinario; negociación).

En relación con el empleo de algunas de las técnicas propuestas, la práctica totalidad de los encuestados afirma que sus unidades realizan, al menos una vez al año, análisis de puestos de trabajo con el propósito genérico de adaptar los recursos humanos a las necesidades organizativas (ajuste de características retributivas a las funciones desempeñadas, previsión de necesidades y cobertura de puestos), intención que sustenta igualmente la contribución de las unidades de recursos humanos a las acciones dirigidas a la reestructuración de sus organismos. A esta actividad de análisis, además de los entrevistados (subdirectores generales), se sumarían subdirectores adjuntos, otros directivos, vocales asesores y jefes de área. Aunque en términos de participación (volumen de actores involucrados) no se detecta descontento, sí es llamativo que la mayoría de los entrevistados declare que el margen de autonomía de que disponen para hacer realidad los cambios que consideran necesarios en relación con la distribución de puestos dentro de su ámbito de competencia sea reducido.

Si bien, desde la vertiente de la valoración psicosocial de las iniciativas adoptadas en pos de una mayor cohesión grupal, resulta meritoria la voluntad de facilitar la integración de los empleados recién incorporados a través de programas de acogida y orientación interna, es decepcionante corroborar que la práctica totalidad de nuestros interlocutores admita ignorar si en algún momento y con qué finalidad se han realizado estudios sobre la cultura y el clima en su Administración. Lo mismo sucede al inquirir si tienen constancia de la realización de estudios sobre motivación: aunque la mayoría considera necesario en el contexto administrativo en el que trabajan la existencia de programas de motivación (que permitan una adecuación retributiva del puesto a las funciones del mismo y que permitan compensar la productividad de forma individualizada) - en algunos casos se informa de que se han adoptado medidas orientadas a la motivación-, la práctica totalidad de los consultados reconoce que no se han llevado a cabo investigaciones.

En lo que atañe a la formación, la mayoría muestra un grado de acuerdo elevado con la política seguida en esta área (a partir de los acuerdos firmados entre Administración y sindicatos en 1994), tanto en las programaciones destinadas a los puestos base, como en las orientadas a las posiciones intermedias o gerenciales (se valoran especialmente las actividades sobre normativa procedimental, tecnologías y presupuestación), y, en consonancia, tampoco detectan ningún déficit digno de mención en la formación del personal de cualquiera de esos niveles de responsabilidad.

En relación con los procesos de evaluación, la totalidad de los entrevistados estima necesaria la existencia en las Administraciones de algún sistema que permita evaluar formalmente el rendimiento de los empleados; la mayoría confirma que se dispone en la actualidad de algún sistema que lo permite hacer de una manera formal y, aunque lo encuentran aceptable, introducirían en él ajustes encaminados a dotarlo de mayor precisión y de una gama de indicadores más objetivos.

En la misma línea, aunque nadie objeta los actuales programas de seguridad y salud laboral, se sugieren, en general, mejoras que deberían confluir sobre los aspectos preventivos. 
En lo que respecta a los procesos de auditoría de la gestión y administración de recursos humanos, el panorama resulta más sombrío: los directivos no reconocen la existencia de procesos transversales formales que a iniciativa de los órganos superiores de la Administración se hayan aplicado en la totalidad de los departamentos y, más bien, se admiten experiencias particulares adoptadas en ministerios concretos con el fin de detectar fallos internos e identificar posibles soluciones. Se trataría, por tanto, de acciones encaminadas al control de procesos que abarcarían aspectos de la función de personal, pero no propiamente de dispositivos ad hoc centrados exclusivamente en la administración, gestión y política de recursos humanos.

Sobre esta última dimensión de la función de personal, los cargos consultados admiten haber participado en experiencias de diseño de políticas de recursos humanos y destacan la motivación de los empleados, el rediseño de la planificación de carreras vinculada a la evaluación del rendimiento, la mejora de la movilidad vertical y la adaptación de los puestos de trabajo a las funciones y a los niveles de responsabilidad como las principales áreas que éstas deberían atender. Sostienen, no obstante, que el escaso margen que los encargados del personal tienen sobre algunas de las dimensiones de la gestión o las «inercias» inherentes a las organizaciones de carácter burocrático, sin duda, seguirán obstaculizando notablemente la elaboración de cualquier política de gestión de recursos humanos en la Administración.

Cuestionados, precisamente, por su grado de participación en relación con algunas actividades que se podrían considerar propias de su campo de trabajo, muestran insatisfacción con su contribución a tareas vinculadas con el estudio de clima y motivación (por ausencia de iniciativas) o la participación en procesos de cambio organizativo y auditoría de personal. Por el contrario, la previsión de necesidades, los procesos de selección y la planificación de la formación son las áreas en las que reconocen sentir mayor satisfacción.

Para finalizar, aunque reconocen que la gestión de recursos humanos ha mejorado en los últimos años en términos de transparencia, seguridad y medios tecnológicos, la mayor dotación de efectivos para las unidades encargadas de esta función, junto con la búsqueda para los años venideros de mayores avances en el diseño y aplicación de los sistemas de evaluación del desempeño, el perfeccionamiento de las acciones formativas y la consecución de mejoras en los mecanismos de coordinación con las unidades de inspección y control de servicios suponen las principales reclamaciones a la hora de evaluar la capacidad actual y futura del departamento.

\section{Función pública en Portugal. Etapas de transformación}

Las supuestas raíces comunes de los sistemas de función pública español y portugués, referidas en la introducción, muestran un marco de empleo similar, ya que como señala França (2003: 1-9) «el régimen del empleo público en Portugal continua siendo un régimen estatutario de carrera con tendencia a lo vitalicio»; evidentemente, en un contexto histórico y territorial, político y, por ende, administrativo diferente. En ellos nos vamos a centrar en las próximas líneas.

Sin pretender valorar más que de soslayo el contexto histórico portugués, y, simplemente, recordando su conexión con el español a través del modelo napoleónico de Administraciones públicas vigente desde principios del siglo XIX, sí procede significar algunos de los procesos de urbanización y socialización vividos por el país vecino desde los años cincuenta como detonantes del nuevo modelo de Estado, que derivaría décadas después y, en consecuencia, del tipo de servicio público que le reclamarían sus ciudadanos: «la larga tradición de un Estado centralizado, en largos períodos autoritario, enraizando la idea de que el Estado y sus agentes se sobreponían a los ciudadanos; la complacencia de estos en relación a quienes ellos suponían dependientes; el bajo nivel de educación generalizado de la población y el diferencial que, a este respecto existía, en relación a los agentes de la Ad- 
ministración; la complicación, sistemáticamente cultivada de las reglas administrativas, que aseguraban el dominio de esos agentes; la reluctancia o, por lo menos, la desconfianza, en aceptar la existencia de órganos de decisión intermedios» (Valente de Oliveira 2001: 18 y 2003: 121-123).

Igualmente, la cuestión territorial marca una clara línea de actuación de los poderes públicos y de sus organizaciones conformando una nueva realidad, que, evidentemente, modifica el sentido mismo de la acción pública y, con ella, el papel y funciones de los empleados al servicio de sus diferentes Administraciones y territorios. Así, para López Mira (2002: 13), Portugal pasa por ser uno de los Estados más antiguos del continente, que es lo mismo que decir del mundo desarrollado «(...) una realidad estatal que posiblemente sea la más antigua de Europa; está lejos de nuestra intención afirmar que la definición de Portugal como Estado se adelantó en varios siglos a las teorizaciones de Bodino, Hobbes, Locke o Montesquieu, pero sí que es un hecho olvidado con frecuencia que, en algunos trazos prototípicos de los modernos Estados-nación, el caso portugués es anterior a las unitaristas Francia, Inglaterra o España del siglo XV; concretamente, el trazado fronterizo que permite delimitar el ejercicio de la noción de soberanía resulta ya de manifiesto en pleno siglo XII, y continuó de forma ininterrumpida prácticamente con los mismos límites hasta nuestros días», además de uno de los más centralizados y centralistas de la actual Europa occidenta ${ }^{17}$. En suma, el devenir histórico desemboca en un modelo de Estado fuertemente centralista, con una escasa descentralización territorial en torno a dos regiones autónomas y varias regiones administrativas (19), pero con una relativa descentralización funcional (18 distritos en el continente y multitud de servicios desconcentrados toda la periferia lusa) y una fuerte planta local (308 municipios y 4.251 freguesias, además de sus correspondientes figuras de asociacionismo local). Obviamente, la reforma administrativa portuguesa tiene que adaptarse a dicho marco, generándose un proceso de difusión que trasmitiría desde el nivel central un modelo que resultaría posteriormente reproducido - con frecuencia, de forma mimética- en su Administración funcional y periférica, regional y local; así, incluso a las regiones con estatuto especial, como las autónomas Azores y Madeira, se les aplica el régimen de funcionarios estatal, por lo menos, en lo que se refiere al personal dirigente y al régimen jurídico del contrato individual de trabajo, si bien con adaptaciones a su propia organización deberá contemplar lo expuesto en la normativa estatal, como veremos en las siguientes líneas.

El contexto político luso, como síntesis de las circunstancias reseñadas, varía sustancialmente, como sabemos, a mediados de la década de los setenta con la transformación de un sistema político dictatorial en otro democrático en virtud de la Revolución de los Claveles (abril de 1974). Desde 1974 a 1976, el sistema político portugués se transforma completamente, consolidando el sistema democrático como ruptura con el sistema corporativo autoritario salazarista. Se da paso al modelo actual portugués de la II República tras los diferentes procesos electorales y la promulgación de la Constitución del proceso revolucionario, de 2 de abril de 1976. Desde entonces hasta nuestros días, nos encontramos ante una Administración pública que contiene un modelo de empleo público de evolución parcial en términos de fines, medios y prácticas ${ }^{18}$ : fruto de la transformación política revolucionaria, de forma similar a España, el marco jurídico modificó algunas reglas del juego administrativo, pero no rompió las inercias de la cultura administrativa, lo que incidió sobre el perfil del trabajador de la Administración portuguesa, que seguirá formando parte de esta estructura organizativa tres décadas después, adaptándose paulatinamente a los cambios programados en los sucesivos gobiernos constitucionales (diecisiete desde 1976) desde la transición hasta nuestros días.

${ }^{17} \ll(\ldots)$ aquel que conserva con mayor pureza los trazos propios de la clásica definición estatal, nacida bajo el paraguas del unitarismo: un solo pueblo, un solo territorio, un solo Derecho, un solo poder, una soberanía única» (López Mira 2002: 13).

${ }^{18}$ Para algunos, «el problema del volumen de efectivos constituye una discusión recurrente de la administración pública portuguesa. Desde el período de la descolonización en que algunos millares de trabajadores públicos de las ex-colonias vinieran a engrosar el número de los ya existentes se está intentando controlar la admisión de nuevos elementos a través de medidas de congelación. Estas medidas fueron siempre, hábilmente o sin pudor, superadas o no aplicadas. La verdad es que a la subida de la media anual de crecimiento de los efectivos (del 1,1\% en 1991 al 2,7\% en el quinquenio siguiente, y al 3\% entre 1996 y 1999) constituye la respuesta a la asunción por el Estado de nuevas tareas en el campo social que no siempre se tradujo en una mejor calidad de los servicios prestados» (França 2003). 
Cómo último aspecto destacado en la conformación de la función pública portuguesa, debemos referirnos a la influencia transnacional de las «recetas de cambio del Estado» (OCDE, Banco Mundial, UE), que suscitarían procesos de reforma y modernización administrativa dotadas de una gran capacidad para modificar sustantivamente el status quo del empleo público luso. Al respecto, algunos autores portugueses (Oliveira 2001: 108; Oliveira 2004: 75-81; Ferraz 2001: 60-63; Ferraz 2004: 83-91) destacan el hecho de que las reformas y modernizaciones emprendidas en todos los países de la órbita de la OCDE hayan resultado similares, estando caracterizadas por las privatizaciones y la desburocratización, la mejora de la relación con el ciudadano, el intento por consolidar una estructura administrativa estable que dirija la modernización, las actuaciones sobre el procedimiento administrativo, la movilidad de los empleados públicos, etc.

Por tanto, los procesos de modernización y/o reforma en Portugal serán los referentes que guíen los cambios sucesivos de un modelo de función pública, que, desde abril de 1974, ha venido implementando procesos de cambio, como veremos, en cuatro etapas: 1974-1985, la reforma administrativa y la Revolución de Abril, de 1974; 1985-1995, revolución managerial de la Administración pública; 1996-2002, reinvención de la Administración pública; y 2002-2005, reingeniería en la Administración pública.

Centrándonos en la función de personal lusa, hay que reiterar el hecho de que las amplias etapas anteriormente descritas implican, además de la creación de marcos normativos e institucionales, una gran carga de transformación del personal al servicio de la Administración pública portuguesa. Desde finales del Estado Novo, pasando por la Revolución de 1974 y el inicio de la democracia, hasta nuestros días, podemos comprobar cómo el número de funcionarios se incrementa en Portugal, desde los 155.213 de 1968 a los 716.418 de 1999 (Oliveira 2004: 77).

Como ya enunciamos, los primeros años de la función pública lusa actual comprenden desde mediados de la década de los setenta -recién iniciado el camino democrático- hasta mediados de los ochenta (1974-1985), momento en el cual se crean las estructuras institucionales de impulso, dirección y gestión de recursos humanos en sus organizaciones públicas centrales. Entre las iniciativas más destacadas del periodo se encuentra la elaboración de un Estatuto de la Función Pública y la creación de la Dirección General de la Función Pública y el Departamento Central de Personal (SCP), posteriormente sustituidos por la Dirección general de Empleo y Formación y la Comisión de Racionalización de Efectivos de la Administración Pública (CREAP). Los marcos normativos se adaptan al nuevo esquema surgido de la Constitución de 1976 (de forma especial, la homologación de leyes sobre el Estatuto de Cuadros Superiores, reclutamiento y sistemas de carrera y régimen disciplinario), poniéndose en marcha las organizaciones de formación e investigación en materia de reforma administrativa y gestión de recursos humanos: Instituto Nacional de Administración (INA) y Centro de Estudios y de Formación Autárquica (CEFA). En paralelo, se introduce un primer Sistema de Evaluación de los Funcionarios (que seguirá presente con más o menos fuerza hasta nuestros días) y se procede a la reestructuración de las carreras de los empleados públicos.

Desde 1985 a 1995, se desenvuelve la segunda etapa de cambio bajo el influjo de la revolución del management en las organizaciones públicas. Asistimos, pues, a una fase caracterizada por el intento de cambio del sistema cultural organizativo basada en los presupuestos del New Public Management ${ }^{19}$. Entre las actuaciones más sobresalientes del momento reformista se podrían reseñar la reforma del sistema retributivo de la función pública, la aprobación del Código de Procedimiento Administrativo, la promulgación de la Ley de los Disponibles (que implicaba la movilidad de funcionarios) y la puesta en marcha de la Carta Deontológica del Servicio Público.

${ }^{19}$ Dicho periodo se podría subdividir en dos subetapas: desde 1986 a 1992, dominada por aspectos de desintervención y desburocratización; y desde 1993 a 1995, centrada en el objetivo de introducir el cambio cultural y la gestión de la calidad en los servicios públicos. 
Ya entrados los años noventa, nos situamos en la tercera etapa de cambio en la función pública, que se despliega hasta los inicios del siglo XXI (1996-2002). Las propuestas de transformación durante este periodo tienen su base -como en casi la totalidad de los países de la OCDE- en los trabajos de Osborne y Gaebler, relativos a la transformación de las organizaciones públicas a través del desarrollo del célebre concepto de «reinvención del gobierno». Es significativo el cambio de sentido en relación a las experiencias gerenciales anteriores, produciéndose un giro en la reforma administrativa y con ella una nueva orientación hacia los recursos humanos, que se traduce en el caso portugués en la revocación del Diploma de los Disponibles; la aprobación del Consejo Superior de Administración y Función Pública; la puesta en marcha de una Auditoría de Recursos Humanos y de Modernización Administrativa; el análisis de la situación social de la función pública a través de la Ley de Balance Social; y la entrada en vigor del Reclutamiento General de la Función Pública, creándose la Inspección General de la Administración Pública, aprobándose el Estatuto del Personal Dirigente y el Instituto de Gestión de la Base de la Datos de los Recursos Humanos de la Administración Pública (IGDAP).

La última etapa de transformaciones de la función pública portuguesa abarca hasta la actualidad (20022005), durante la cual las dificultades financieras del país y los compromisos adquiridos en materia de reforma administrativa con la Unión Europea (principal financiadora del proceso, pero vigilante de su cumplimiento a través de la Estrategia de Lisboa y el Pacto de Estabilidad acordado por los Estados miembros) contextualizan los cambios de una concepción de la reforma, que transita desde la idea de reinvención a la de reingeniería -modelo que algunos denominan gestionarial (Oliveira 2000: 6-16). En este escenario, la llegada al poder de la coalición de centro-derecha, que derrotó a los socialistas en 2002 y que se mantuvo en el poder hasta 2005, alteró sustancialmente la filosofía de la reforma administrativa, orientándola hacia medidas de inspiración neoliberal, que influirían en la promoción de prácticas gerenciales como la dirección por objetivos o la difusión de sistemas de evaluación del desempeño (efectuada tanto individualmente como a nivel de unidad). En este escenario, la justificación de la adopción de medidas radicales de recorte del Estado es ilustrado elocuentemente por França (2003): «el consumo público sobrepasa el 51\% do producto interno; los gastos en función pública representan el 15\% do PIB habiendo aumentado de 1995 a 2001 con motivo del reflejo excesivo en la masa salarial de los aumentos de jubilaciones y automatismos de progresión en las categorías y en los salarios; la cuota de gasto público municipal es de apenas el 9\%; el 79,3\% de los funcionarios públicos integran la Administración central del Estado; el 25\% de los trabajadores de la función pública desempeñan funciones en el distrito de la capital nacional; (...) si el ratio del empleo público sobre el empleo total $(15 \%)$ no es particularmente negativo en términos comparativos con otros países europeos no puede dejar de comentarse que hasta el 2002 el empleo público no dejó de crecer (3,6 veces en 32 años); preocupantes son los valores relativos la estructura de edad donde cerca del 40\% de los funcionarios integran los niveles por encima de los 45 años; más preocupante es el peso de los funcionarios con titulaciones iguales o inferiores a los 4 años de escolaridad (18,9\%) o iguales o inferiores a los 9 años $(42,8 \%) »$.

El primer documento político de importancia relativo a la política de gestión pública de esta etapa se presentó en junio de 2003, y se denominó Líneas de Orientación para la Reforma de la Administración Pública. Con posterioridad se han adoptado una serie de resoluciones jurídicas que han dado sentido normativo al cambio político: es el caso de la Resolución del consejo de Ministros n. ${ }^{\circ}$ 53/2004, de 21 de abril, en la que se incluyen medidas de gran calado en relación a los empleados públicos, o la modificación de las condiciones de acceso al empleo público y con ellas el impulso del nuevo «régimen de contrato individual» (Ley n. ${ }^{\circ} 23 / 2004$, de 22 de junio) ${ }^{20}$, que limita la actuación de los funcionarios a actividades básicas de soberanía, externalizando la mayor parte de actuaciones públicas.

\footnotetext{
${ }^{20}$ «Esta modalidad de empleo, que no se aplica a las actividades que impliquen el ejercicio de poder de autoridad o el ejercicio de poder de soberanía, permite la contratación a través de un proceso de reclutamiento y selección transparente y simplificado, garantizando los principios de la publicidad y de la imparcialidad» (Ferraz 2004: 88).
} 
Siendo prematuro avanzar conclusiones sobre esta última y más reciente etapa, sí es posible prever que los próximos años no alumbrarán más procesos de modernización que los derivados de las iniciativas de reducción del déficit público ${ }^{21}$, dando continuidad a algunas de las experiencias de gestión más significativas que se pusieron en marcha en los últimos años (evaluación del desempeño y laboralización de la función pública) y a la aplicación de técnicas de gestión ya reconocidas -y apoyadas por la Unión Europea-, implementadas en determinadas unidades estratégicas.

En resumidas cuentas, a lo largo de estos más de treinta años de transformaciones del sector público portugués los numerosos intentos de cambio han arrojado «luces y sombras» (Oliveira 2001: 93-119), que, para bien y para mal, se han proyectado de una forma especialmente manifiesta sobre el empleo público luso.

\section{La integración de los empleados públicos en los procesos de reforma portugueses}

En relación con el papel desempeñado por parte de los empleados públicos en el proceso de cambio portugués, Oliveira (2001: 114-115) señala que en los primeros diez años de la reforma administrativa apenas se contó con la implicación de los funcionarios en dicho proceso, lo que en cierto modo supuso un freno a sus posibles avances: «la implicación de los funcionarios públicos estuvo ausente de la estrategia de modernización administrativa, más allá de que como se afirma en el Informe de la Comisión para la Calidad y Modernización de la Administración Pública el poder político no concedió autonomía y poder efectivo a los gestores profesionales de la Administración Pública». Además las experiencias de gestión, sus nuevos modelos y técnicas chocaban con una organización basada en parámetros y valores burocráticos, de corte legalista, y donde el «muro de la norma» mitigaba los esfuerzos de cambio (Ferraz 2004: 88).

Tras los intentos precedentes, los Gobiernos socialistas (1995-2002) aplicaron a la gestión de personal una estrategia de no confrontación, intentando incorporar a la reforma administrativa a los empleados públicos (Oliveira 2001: 119).

El cambio de Gobierno de 2002, y con él la orientación de la reforma de corte «reingenieril», han imprimido un nuevo giro a la reforma administrativa y a la implicación de los funcionarios, evolucionando desde la primigenia construcción social de la Administración pública, con medidas orientadas a «dignificar» y a consolidar el empleo público (Ferraz 2004: 88), hacia la flexibilización y la laboralización de la gestión del personal ${ }^{22}$, que prosigue -a juzgar por el mantenimiento a principios de 2006 de normas a través de las cuales el Gabinete preceden-

${ }^{21}$ Si observamos la ubicación de la Secretaria de Estado que en esta fecha impulsa el proceso (dentro del Ministerio de Finanzas y Administración Pública) vemos, y así nos lo trasladó su titular, que la prioridad reformadora es el saneamiento de las cuentas públicas. A lo largo de la entrevista, se planteó una especie de silogismo según el cual la Administración pública (y con ella su personal, dada la evidente carga financiera de los sueldos de los empleados en los presupuestos de las organizaciones públicas) debe reducir el déficit y esto implica congelar salarios, aumentar la edad de jubilación, para posteriormente asignar mejor los recursos, simplificar procedimientos, mejorar técnicas, reordenar la planta territorial de la Administración central, proceder a la descentralización administrativa y posteriormente a la política (con base en la legitimidad democrática). Estas conclusiones se extraen de una serie de entrevistas realizadas el 25 de mayo de 2005 a los siguientes responsables políticos de la Administración central portuguesa (a los cuales agradecemos especialmente su interés y el tiempo dedicado a nuestra investigación): Sr. João Figueiredo (Secretario de Estado de Finanzas y Administración Pública), Sr. Eduardo Cabrita (Secretario de Estado Adjunto de Administración Local) y Sr. Luis Valadares Tavares (Presidente del INA).

${ }^{22}$ El debate sobre la forma predominante de los contratos que vinculan al empleado con la Administración Pública es similar en España y Portugal y se mueven en torno a las propuestas de «laboralización» vs. «estatutarización» («contratualização» vs. «estaturização»), aunque para el caso portugués resulta diferente no sólo por la cantidad de funcionarios sino también por el escaso número de puestos de carácter laboral: en 1996 el $91 \%$ de los 600.000 empleados públicos eran estatutarios y el resto contratados (Nabais 2001: 65-68). 
te consolidaba la transición del sistema estatutario al laboral de forma paulatina, selectiva pero inexorable $\mathrm{e}^{23}$ durante el mandato del actual Gobierno socialista: «Con excepción de algunos grupos profesionales en razón de la materia (por ejemplo, diplomacia, investigación, magistratura judicial y del Ministerio Público, cuadros permanentes superiores de las fuerzas armadas y de la seguridad, etc.) y otros en razón de su especificidad y necesidad (por ejemplo en el área de la salud) se entiende que la generalidad de las profesiones de la administración pública pueden ser desempeñadas en régimen de contrato individual de trabajo» (França 2003). En cualquier caso, no se consigue la deseada implicación de los funcionarios, sino todo lo contrario, dado que las medidas propuestas por el Gobierno, entre otras razones, acentúan la politización de la administración (sobre todo de los dirigentes) ${ }^{24}$.

\section{Portugal y los nuevos retos para una vieja función pública}

En materia de reforma administrativa, la evolución de la Administración pública portuguesa ha caminado en paralelo a los programas llevados a cabo en la mayor parte de los países de la OCDE. A lo largo de estos últimos treinta años, Portugal ha tenido que adaptar sus estructuras administrativas al nuevo régimen democrático; asumir el reto de la reforma y la modernización de sus organizaciones públicas; ingresar como miembro de pleno derecho en la Unión Europea; y mantener los criterios de estabilidad presupuestaria que marcan los nuevos «gobernantes» mundiales (OCDE, Banco Mundial, FMI...). En definitiva, muchos retos para gran cantidad de límites económicos, sociales, políticos y administrativos.

La función pública ha constituido una de las variables independientes de mayor impacto estratégico sobre el funcionamiento de las organizaciones públicas portuguesas, pero, a la vez, ha operado como elemento dependiente en su exposición a las diferentes iniciativas de modernización y cambio que los distintos gobiernos lusos han venido promocionando en las tres últimas décadas. Los resultados en materia de reforma administrativa son muy limitados, y en lo tocante a la función pública aún más. Ni siquiera las corrientes teóricas de gestión pública contemporáneas más influyentes o los poderosos procesos de difusión de las nuevas tecnologías de la Información han podido vehiculizar los cambios que son deseables en materia de función pública.

En Portugal, entendemos que no se ha logrado el cambio necesario en la función de personal, entre otras razones, porque, nuevamente, lo hard se ha impuesto a lo soft, la perspectiva reingenieril a la de las relaciones humanas: a lo largo de estas últimas décadas los cambios más profundos en las organizaciones públicas han provenido de los elementos tecnológicos que dan apoyo a la gestión, al trabajo administrativo, a la toma de decisiones, etc., quedando a un lado el factor humano, el desarrollo de sus competencias; su capacidad de trabajo en equipo; la mejora de las relaciones en las unidades administrativas; los componentes emocionales de los sistemas de compensación; o su implicación en los procesos de cambio. En suma, aspectos de cultura administrativa (Ramió 1999: 37-38; Neves 2003: 167-179), que en su desatención se revelan como los principales escollos de la carrera por mejorar las capacidades y los resultados de las Administraciones públicas.

Francamente, en relación con la modernización de los recursos humanos de la organización central portuguesa, no se puede concluir que nos encontremos todavía en un punto de partida. La implementación de programas de evaluación, de atención al ciudadano, de mejora de los servicios on-line a través de internet niegan categóricamente tal dictamen. Sin embargo, los gobernantes se han mostrado incapaces de abordar convincentemente los programas orientados a la transformación de la función de personal que han propuesto a lo largo de estas tres

\footnotetext{
${ }^{23}$ Ley n. ${ }^{0}$ 23/2004, de 22 de junio, que aprueba el régimen jurídico del contrato individual de trabajo de la Administración pública.

${ }^{24}$ Ley n. ${ }^{\circ} 2 / 2004$, de 15 de enero, que aprueba el estatuto del personal dirigente de los servicios y organismos de las Administraciones central, local y regional del Estado.
} 
últimas décadas ${ }^{25}$ y, lo que es peor, a menudo han atribuido injustamente al funcionariado el origen de los males sociales y económicos del país, terminando por diseñar políticas de recursos humanos de manifiesto afán ejemplarizante en las que actuaciones punitivas basadas en el recorte de las prestaciones sociales, la congelación salarial o el aumento de los controles internos amenazan con hipotecar por completo el apoyo y la participación de los empleados públicos en cualquier programa venidero de reforma.

\section{Conclusiones: Caminos paralelos, deficiencias comunes}

Como hemos podido ver, una revisión detallada de documentación relacionada con las políticas de gestión pública emprendidas por las Administraciones centrales española y portuguesa, aconseja una redefinición de las líneas de actuación en materia de función de personal, siempre en función de sus particulares necesidades de cambio.

En el caso español se ha dado continuidad a un camino parcialmente abandonado hace más de dos décadas a través de las reformas del modelo de recursos humanos, cuyo penúltimo capítulo, pero no por ello menos destacado, supone la aprobación del Estatuto Básico del Empleado Público.

En el caso portugués, y en la misma línea, encontramos una continuidad de los modelos de nueva gestión pública de los recursos humanos acometidos por gobiernos de diferente signo político, desde los años 90 hasta nuestros días.

Las raíces normativas y culturales napoleónicas, que en ambos casos les han dado sentido, aún se mantienen firmes, de forma que los cambios producidos a través de los procesos de reformas-modernizaciones tienen impactos limitados sobre los elementos estratégicos de gestión de recursos humanos en sus unidades centrales de gobierno. Todo ello pese a las sucesivas recomendaciones de algunos organismos internacionales de referencia en materia de modernización de las Administraciones públicas, que ponen de manifiesto la necesidad de profundizar en mecanismos más dinámicos de gestión del personal tales como el mantenimiento del personal altamente cualificado, la adopción de medidas de «rejuvenecimiento de la plantilla», o la introducción de elementos de conciliación de la vida laboral y familiar dentro de las estructuras de personal de las organizaciones públicas. Estos elementos chocan con los tradicionales de la dirección de personal, sometidos a cierta ortodoxia de gestión por parte de los organismos de intervención de personal (inspección de servicios y similares, además de los tradicionales límites que impone el Departamento de Economía) de las estructuras centrales de recursos humanos, que constriñen las capacidades de maniobra de los gestores de cada uno de los departamentos sectoriales, y limitan sus posibilidades de dirección, sobrecargando, en algunos casos, las unidades administrativas a través del recurso a la subcontratación del personal.

A modo de cierre, podríamos destacar que, efectivamente, los caminos en materia de gestión de recursos humanos en las Administraciones centrales española y portuguesa en estas últimas dos décadas, han discurrido de forma paralela, con deficiencias comunes, pero en escenarios y marcos socioeconómicos tan diferentes que los modelos político-administrativos resultantes suponen la consolidación de sistemas de empleo público distintos. En la Administración General del Estado en España, con una orientación clara hacia organizaciones que buscan consolidar estructuras de empleo público, rejuvenecer plantillas o mejorar algunas de las condiciones sociolaborales de sus empleados. En la Administración central portuguesa, hacia modelos más neoempresariales que implican reducción de plantilla, laboralización del personal y el auxilio a través de fórmulas de organización más próximas a las sociedades privadas.

${ }^{25}$ Son muy ilustrativos los comentarios de Ferraz (2001: 60-63), siguiendo a Caiden (1991), quien señala que «(..) la reforma administrativa tiene un lugar permanente en la Administración Pública y una prioridad cada vez mayor en el Gobierno, no sólo por causa de la mejora de la actuación administrativa sino para que los mejores gobiernos puedan realizar el sueño y las ambiciones del hombre. Ella se debe convertir en una actividad continua y no meramente en un evento periódico al cual los gobiernos recurren en situaciones de ruptura». 
En ambos casos aún queda mucho camino por recorrer, sobre todo en relación con los modelos de gestión estratégica de recursos humanos, la mejora de la dimensión psicosocial de la función de recursos humanos (factores de motivación, cultura y clima laboral), los sistemas de evaluación del desempeño del personal, las estructuras de retribución vinculadas a estos sistemas o el desarrollo de una nueva función directiva y semidirectiva basada en esquemas de competencias que determinen la calidad de la gerencia pública. Porque entendemos que no es posible introducir mecanismos de mejora del desempeño y desarrollo personal (formación, diseño de carrera, evaluación de la actuación, compensación), sin definir previamente el rol que en todo ello debe reservarse al ejercicio del liderazgo público. Más allá de los recursos (normativos, institucionales, económicos, materiales o tecnológicos) que se pongan al servicio de la función de recursos humanos, el progreso de esta área dependerá de la habilidad política y de la alta dirección pública para que el compromiso de las personas con la organización, como valor cultural-cardinal, sea compartido a todos los niveles e infunda la orientación de cada iniciativa administrativa.

\section{REFERENCIAS BIBLIOGRÁFICAS Y DOCUMENTALES}

Cavero, I. y Zamora, T. (1996), Los sistemas políticos. Madrid: Universitas.

CIS (1983), «Los funcionarios ante la reforma de la Administración» (Estudio n. ${ }^{\circ}$ 1359). Mimeo.

- (1988), «Actitudes y opiniones de los funcionarios de la Administración civil del Estado» (Estudio n. ${ }^{\circ}$ 1741). Mimeo.

- (1990), «Opiniones y actitudes de los funcionarios en torno a la función pública» (Estudio n. ${ }^{\circ}$ 1871). Mimeo.

- (1993), «Imagen social de los funcionarios» (Estudio n. ${ }^{\circ}$ 2054). Mimeo.

- (1999a), Datos de Opinión-Boletín CIS n. ${ }^{\circ} 20$.

- (1999b), Datos de Opinión-Boletín CIS n. ${ }^{\circ} 21$.

- (2000), Datos de Opinión-Boletin CIS n. 26.

- (2003), Datos de Opinión-Boletín CIS n. ${ }^{\circ} 31$.

Colomer, J. M. (1995), «España y Portugal: Regímenes de liderazgo de partido» en J. M. Colomer, dir., La política en Europa. Introducción a las instituciones de quince países. Barcelona: Ariel.

Comisión para el estudio y preparación del Estatuto Básico del Empleado Público (2005), Estatuto Básico del Empleado Público. Informe de la Comisión. Abril 2005, Goberna: Centro de Nuevas Estrategias de Gobernanza Pública. Madrid: INAP.

Domínguez, L. y Venade, N., coords., (2004), As Eurorrexións e o futuro de Europa: O Modelo da Eurorrexión Galicia-Norte de Portugal. Porto: Eixo Atlántico do Noroeste Penínsular.

Ferraz, J. F. (2001), «Considerações sobre o conceito de reforma administrativa», Revista de Administração e Políticas Públicas 2: 60-63.

Ferraz, J. F. (2004), «A reforma administrativa em Portugal: em busca de um novo paradigma», Revista de Pensamento do Eixo Atlántico 6: 83-91.

França, J. A. (2003), «Reforma e inovação em épocas de escassez aguda de recursos: a experiencia portuguesa em curso». VIII Congreso Internacional del CLAD sobre la Reforma del Estado y de la Administración Pública. Panamá, 28-31 Octubre de 2003, pp. $1-9$ (en actas del Congreso).

González, J. C. (1999), Desarrollo político y consolidación democrática en Portugal (1974-1998). Madrid: CIS y Universidad de Salamanca. 
González, J. C. (1985), «Portugal» en J. Ferrando Badía, coord., Regímenes políticos actuales. Madrid: Tecnos.

INAP (1996), La Misión del INAP. Modernización y Cambio en las Administraciones Públicas. Madrid: INAP

INAP/Goberna (2005), Estatuto Básico del Empleado Público. Informe de la Comisión (Abril 2005). Madrid: INAP y Centro de Nuevas Estrategias de Gobernanza Pública. Mimeo.

Lagares, N. (2000), «El sistema político de Portugal» en M. Alcántara, ed., Sistemas políticos de la Unión Europea. Valencia: Tirant lo Blanch.

López Mira, Á. X. (2002), Portugal: a rexionalización imposible. Vigo: Universidade de Vigo.

MAP (2000), Libro Blanco para la Mejora de los Servicios Públicos. Madrid: MAP.

- (2005a), Plan de Racionalización y Rejuvenecimiento de la Administración General del Estado. Madrid: MAP. Mimeo.

- (2005b), «Gestión de Recursos Humanos en las Administraciones Públicas». Documento de trabajo de la Jornada sobre Recursos Humanos en las Administraciones Públicas. Sociedad de la Información. Madrid, 16 de junio de 2005. Mimeo.

MAP/Fundación Formación y Tecnología (1992), La Formación de Directivos para la Modernización de la Administración Pública. Madrid: MAP/Fundación Formación y Tecnología.

Nabais, J. (2001), «O capital humano na Função Pública. Contratualização / Estaturização» en VV.AA., A Administração Pública no limiar do Século XXI: os grandes desafios. Oeiras: INA.

Neves, A. (2003), «Cultura de gestão na Administração Pública» en VV.AA., A reinvenção da função pública. Da burocracia à gestão. Oeiras: INA.

Oliveira, J. A (2001), Gestão Pública e Modernização Administrativa. Oeiras: INA.

Oliveira, J. A. (2004), «A gestão pública na Região do Norte de Portugal», Revista de pensamento do Eixo Atlántico, 6: 75-81.

Parrado, S. (2002), Sistemas administrativos comparados. Barcelona: Editorial Tecnos y Universitat Pompeu Fabra.

Ramió, C. (1999a), «La innovación en la gestión pública desde las corrientes neoempresariales versus la innovación en la gestión pública desde las corrientes neopúblicas: cultura administrativa, valores públicos y credibilidad social: un planteamiento radical». IV Congreso Español de Ciencia Política y de la Administración, de la Asociación Española de Ciencia Política y de la Administración (Aecpa), Granada, 30 septiembre - 2 octubre de 1999 (en actas del Congreso).

Ramió, C. (1999b), Teoría de la Organización y Administración Pública. Madrid: Editorial Tecnos y Universitat Pompeu Fabra.

Román, P. (1993b), «Portugal» en R. Cotarelo et. al., Sistemas politicos de la Unión Europea. Con inclusión de Estados Unidos y Japón. Madrid: Universitas.

Román, P. y Láiz, C. (2003), Política Comparada. Madrid: McGraw-Hill.

Santamaría, J., comp., (1982), Transición a la democracia en el Sur de Europa y América Latina. Madrid: CIS.

Valente de Oliveira, L. (2001), «Os condicionamentos em Portugal» en VV.AA., A Administração Pública no limiar do Século XXI: os grandes desafios. Oeiras: INA.

Valente de Oliveira, L. (2003), «Sociedade, estado e serviço público» en VV.AA., A reinvenção da função pública. Da burocracia à gestão. Oeiras: INA. 